PAPER

\section{In pursuit of barrierless transition metal dichalcogenides lateral heterojunctions}

To cite this article: Yierpan Aierken et al 2018 Nanotechnology 29295202

View the article online for updates and enhancements.

\section{Related content}

\begin{tabular}{l} 
- A theoretical study of the electrical contact \\
between metallic and semiconducting \\
phases in monolaver MoS2 \\
Wendel S Paz and J J Palacios \\
- Electronic properties and transistors of the \\
NbS2-MoS2-NbS2 NR heterostructure \\
Qi Liu, Fangping Ouyang, Zhixiong Yang \\
et al. \\
- Spin polarized vertical transport in stacked \\
\hline TMDCs hetero-junctions \\
A L M T Costa, F W N Silva and E B \\
Barros
\end{tabular}

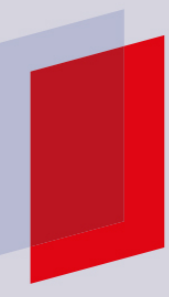

\section{IOP ebooks}

Bringing you innovative digital publishing with leading voices to create your essential collection of books in STEM research. Start exploring the collection - download the first chapter of every title for free. 


\title{
In pursuit of barrierless transition metal dichalcogenides lateral heterojunctions
}

\author{
Yierpan Aierken $^{1}$, Cem Sevik ${ }^{2}$, Oğuz Gülseren ${ }^{3}$, François M Peeters ${ }^{1}$ and \\ Deniz Çakır ${ }^{1,4}$ (1) \\ ${ }^{1}$ Department of Physics, University of Antwerp, Groenenborgerlaan 171, B-2020 Antwerpen, Belgium \\ ${ }^{2}$ Department of Mechanical Engineering, Faculty of Engineering, Anadolu University, Eskisehir, TR \\ 26555, Turkey \\ ${ }^{3}$ Department of Physics, Bilkent University, Bilkent, Ankara 06800, Turkey \\ ${ }^{4}$ Department of Physics and Astrophysics, University of North Dakota, Grand Forks, North Dakota 58202, \\ United States of America \\ E-mail: deniz.cakir@und.edu
}

Received 13 February 2018, revised 23 April 2018

Accepted for publication 1 May 2018

Published 18 May 2018

\begin{abstract}
There is an increasing need to understand interfaces between two-dimensional materials to realize an energy efficient boundary with low contact resistance and small heat dissipation. In this respect, we investigated the impact of charge and substitutional atom doping on the electronic transport properties of the hybrid metallic-semiconducting lateral junctions, formed between metallic (1T and $1 \mathrm{~T}_{d}$ ) and semiconducting $(1 \mathrm{H})$ phases of $\mathrm{MoS}_{2}$ by means of first-principles and non-equilibrium Green function formalism based calculations. Our results clearly revealed the strong influence of the type of interface and crystallographic orientation of the metallic phase on the transport properties of these systems. The Schottky barrier height, which is the dominant mechanism for contact resistance, was found to be as large as $0.63 \mathrm{eV}$ and $1.19 \mathrm{eV}$ for holes and electrons, respectively. We found that armchair interfaces are more conductive as compared to zigzag termination due to the presence of the metallic Mo zigzag chains that are directed along the transport direction. In order to manipulate these barrier heights we investigated the influence of electron doping of the metallic part (i.e. $1 \mathrm{~T}_{d}-\mathrm{MoS}_{2}$ ). We observed that the Fermi level of the hybrid system moves towards the conduction band of semiconducting $1 \mathrm{H}-\mathrm{MoS}_{2}$ due to filling of $4 d$-orbital of metallic $\mathrm{MoS}_{2}$, and thus the Schottky barrier for electrons decreases considerably. Besides electron doping, we also investigated the effect of substitutional doping of metallic $\mathrm{MoS}_{2}$ by replacing Mo atoms with either Re or Ta. Due to its valency, Re (Ta) behaves as a donor (acceptor) and reduces the Schottky barrier for electrons (holes). Since Re and Ta based transition metal dichalcogenides crystallize in either the $1 \mathrm{~T}_{d}$ or $1 \mathrm{~T}$ phase, substitutional doping with these atom favors the stabilization of the $1 \mathrm{~T}_{d}$ phase of $\mathrm{MoS}_{2}$. Co-doping of hybrid structure results in an electronic structure, which facilities easy dissociation of excitons created in the $1 \mathrm{H}$ part.
\end{abstract}

Keywords: first-principles calculations, $\mathrm{MoS}_{2}$, metal-semiconductor interface, Schottky barrier (Some figures may appear in colour only in the online journal)

\section{Introduction}

Heterostructures are the essential components of a wide range of solid-state devices, such as transistors, solar cells, and sensors $[1,2]$. They are fabricated by combining different type of materials, e.g. metal, semiconductor, and insulator. Therefore, the physical properties of the combined system are enhanced or become more controllable as compared to that of each material individually. These tailored properties are strongly related to the interface of two different materials where all interesting and new phenomenon occur. However, along with the emergence of nanostructured materials, dimensionality has become another major factor affecting the physical properties of materials and devices along with the 


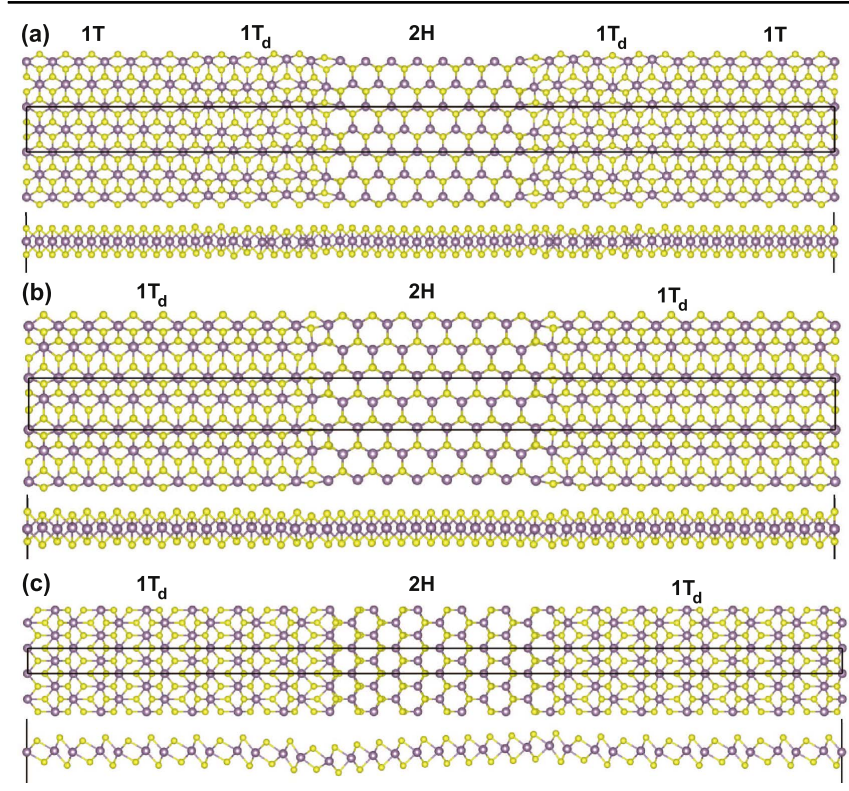

Figure 1. Device models where $1 \mathrm{H}$ phase of $\mathrm{MoS}_{2}$ is sandwiched between metallic $\mathrm{MoS}_{2}$ electrodes. In (a) the $\alpha$-device, (b) the $\beta$-device, and (c) the $\gamma$-device. For the $\alpha$ and $\beta$ devices, the interfaces between metallic and semiconducting $\mathrm{MoS}_{2}$ have an armchair termination while a zigzag termination in the $\gamma$-device.

interface. Thus, solid-state device fabrication with heterostructures based on low dimensional nanomaterials has attracted significant attention and a new research area in material design has been initiated where researchers are expecting unprecedented results, phenomenon and physics [3-5]. Indeed, several advantages of two-dimensional (2D) phase engineering over the three dimensional counterpart has already been demonstrated [6].

In low dimensional heterostructure device architectures, there are usually two types of interfaces connecting different materials: top contact (vertical) and edge contact (lateral) [7]. In top contacts, an overlapping portion of two materials are glued together mainly via interlayer van der Waals (vdWs) interaction, while in edge contacts one dimensional edges of two materials are contacted with covalent bonds without overlapping. The vdWs interaction in top contact introduces a potential gap between the two layers which electrons have to tunnel through, and resulting in higher resistance due to the reduced carrier transmission probability. Naturally, this resistance is much lower in edge contacts owing to the formation of covalent bonds that provides a path for carriers to travel across the interfaces [8,9]. Recently, Eda et al has discovered the coexistence of multi-phase $\mathrm{MoS}_{2}$ that is a promising material for heterostructure device fabrication due to their natural metal-semiconductor-metal structure with clear edge contacts [10]. Considering the distinct electronic nature of these phases, physical properties of these heterostructures [11, 12] can be tuned by phase engineering and novel solid-state device architectures can be realized for several different future applications.

The same research group has synthesized 2D semiconducting heterostructure devices [13, 14] by using metal contacts. As a result of their experimental analysis, they have particularly pointed out the vital importance of the geometry and electronic nature consistency between the metal contact and the heterostructure on the device performance $[10,15]$. Considering this fact, Kappera et al [11] have locally induced $1 \mathrm{~T}$ metallic phase of $\mathrm{MoS}_{2}$ in the $1 \mathrm{H}$ semiconducting phase of it, and they measured that the edge resistance was lower than that of metal contacts by more than a factor of two. Subsequently, $1 \mathrm{~T} \mid 1 \mathrm{H}$ lateral heterostructure has been drawn peculiar attention as a promising contact structure having a higher carrier injection rate. Different arrangements of the interfaces between $1 \mathrm{~T}$ and $1 \mathrm{H}$ phases was investigated through theoretical calculations [16, 17] and the structure formed by the connection of armchair edges of $1 \mathrm{~T}$ and $1 \mathrm{H}$ phases has been determined as an energetically more favorable configuration. However, in these calculations, the more stable metallic structure $\left(1 \mathrm{~T}_{d}\right)$, which arises with small distortion of $1 \mathrm{~T}$ phase, was considered.

The use of metallic TMDCs as metal electrodes are expected to offer a breakthrough in the semiconductor industry as they have negligible heat dissipation and therefore are energy efficient. Among metallic TMDCs, metallic phases of $\mathrm{MoS}_{2}$ (1T- and $1 \mathrm{~T}_{d}-\mathrm{MoS}_{2}$ ) have attracted a growing interest due to its smooth interface with the semiconductor phase of $\mathrm{MoS}_{2}\left(1 \mathrm{H}-\mathrm{MoS}_{2}\right)$. However, $1 \mathrm{H}$ phase is thermodynamically more stable than both $1 \mathrm{~T}$ and $1 \mathrm{~T}_{d}$ phases. Therefore, the stabilization of $1 \mathrm{~T}$ and $1 \mathrm{~T}_{d}$ over $1 \mathrm{H}$ phase becomes an essential requirement for the successful experimental realization of device configurable structures such as $1 \mathrm{~T} / \mathrm{T}_{d}-\mathrm{MoS}_{2} \mid 1 \mathrm{H}-\mathrm{MoS}_{2}$. On the other hand, $1 \mathrm{~T}-\mathrm{MoS}_{2}$ is the meta-stable and undergoes a Peierls transition to a low-energy state $1 \mathrm{~T}_{d}$ (or distorted 1T) and thus, metal contacts with the $1 \mathrm{~T}_{d}$ structure are more stable than the one with the $1 \mathrm{~T}$ phase. However, the $\mathrm{MoS}_{2} 1 \mathrm{~T}_{d}$ phase retransforms to the $1 \mathrm{H}$ phase at room temperature. As far as the relative stability is considered, choosing $1 \mathrm{~T}_{d}$ as metal contact further stabilized the junction. Therefore, understanding the effect of different physical mechanisms on the stability of multiple phases $(\mathrm{H}, \mathrm{T}$, $\mathrm{T}_{d}$ ) of this material is of vital importance to develop a proper control on phase transitions. To this end, we mainly focus on the effect of doping (either charge or atom) on the stability, electronic and transport properties of $1 \mathrm{~T} / \mathrm{T}_{d}-\mathrm{MoS}_{2} \mid 1 \mathrm{H}-\mathrm{MoS}_{2}$ interfaces.

The present paper aims to investigate the electronic transport properties of $\mathrm{MoS}_{2}$ multi-phase lateral junctions when the more stable metallic phase of $\mathrm{MoS}_{2}$ (i.e. $1 \mathrm{~T}_{d}$ ) acts as the contact, which is compared with the $1 \mathrm{~T}$ phase. Further to this, the paper mainly focuses on the effect of doping on the electrical transport properties. In the results section, we first construct three junction models and calculate their transmission without external bias. Then we calculate the electronic properties for different level of doping.

\section{Computational details}

The presented first-principles calculations are based on density function theory as implemented in the Vienna $a b$ initio Simulation Package (VASP) [18-21]. A plane-wave basis set 
based on the projected augmented wave method [22, 23] are used to describe the wave functions. The cutoff energy of the basis is set to $400 \mathrm{eV}$. Exchange-correlation interactions are treated with the generalized gradient approximation (GGA) within the Perdew-Burke-Ernzerhof (PBE) formulation $[24,25]$. A $25 \times 25 \times 1 k$-points mesh is used to sample the Brillouin-zone for monolayer structures of $1 \mathrm{H}, 1 \mathrm{~T}$ and $1 \mathrm{~T}_{d}$ $\mathrm{MoS}_{2}$ and a $9 \times 1 \times 1 k$-points grid is used for the structures shown in figure 1 . A vacuum space of $15 \AA$ is incorporated to avoid interaction between the periodic images. The energy convergence criterion for the self-consistent calculations is set to $10^{-5} \mathrm{eV}$, while the force convergence criterion for the ionic steps is set to $10^{-2} \mathrm{eV}^{-1}$.

Electronic transport across the $1 \mathrm{~T}_{d} / 1 \mathrm{~T}-\mathrm{MoS}_{2} \mid 1 \mathrm{H}-\mathrm{MoS}_{2}$ interfaces is calculated using the self-consistent non-equilibrium Green's functions technique as implemented in TranSIESTA [26] which is interfaced with the SIESTA code [27]. Double-zeta (plus polarization) numerical orbital basis sets are used for all atoms. We employed norm-conserving pseudo-potentials [28], the GGA/PBE functional, and an energy cutoff for the real-space mesh of $250 \mathrm{Ry}$. In order to get accurate transmission spectra, the 2D Brillouin-zone normal to the transport direction is sampled by meshes composed of $100 \mathrm{k}$-points in the periodic direction. While the SIESTA code uses a localized basis set and norm-conserving pseudo-potentials, the calculated lattice parameters for different phases of $\mathrm{MoS}_{2}$ agree well with those obtained from the VASP code.

\section{Results}

We first calculate the structural properties of $1 \mathrm{H}, 1 \mathrm{~T}$ and $1 \mathrm{~T}_{d}$ phases of $\mathrm{MoS}_{2}$. For the $1 \mathrm{~T}$ and $1 \mathrm{H}$ hexagonal unit cells, the optimized in-plane lattice constant is obtained as $3.18 \AA$. On the other hand, the optimized lattice constants are $a=3.18 \AA$ and $b=5.72 \AA$ for the tetragonal $1 \mathrm{~T}_{d}$ unit cell. These values are in good agreement with previous calculations [29]. It was previously discovered the coexistence of $1 \mathrm{~T}_{d}$ phase with other two phases indicating their experimental stabilities, yet it is also possible to relax the $1 \mathrm{~T}_{d}$ phase to to $1 \mathrm{~T}$ phase using external source, such as electron beam irradiation [10]. In experiment, $1 \mathrm{~T}$ and $1 \mathrm{~T}_{d}$ are indistinguishable, because the $\mathrm{S}$ atoms are the same in two cases, only the Mo form cluster which STM image is limited to differentiate.

Next, we systematically investigate the electronic and transport properties of three different device architectures, called as $\alpha, \beta$, and $\gamma$, denoted in figure 1 . In all device models, the semiconducting $1 \mathrm{H}-\mathrm{MoS}_{2}$ phase is sandwiched between two $1 \mathrm{~T}_{d}$ metal electrodes to create Schottky contacts at the interfaces. In the $\alpha$ structure, the metallic part consists of both $1 \mathrm{~T}$ and $1 \mathrm{~T}_{d}-\mathrm{MoS}_{2}$ phases. The size of metallic and semiconducting parts are larger than $20 \AA$ along the transport direction. The interface between the $1 \mathrm{~T}_{d}-\mathrm{MoS}_{2}$ and $1 \mathrm{H}-\mathrm{MoS}_{2}$ phases have either an armchair termination as in the case of the $\alpha$ and $\beta$ structures, or a zigzag termination as in the case of the $\gamma$ structure in order to investigate the influence of the contact type on the calculated properties. We predict that the

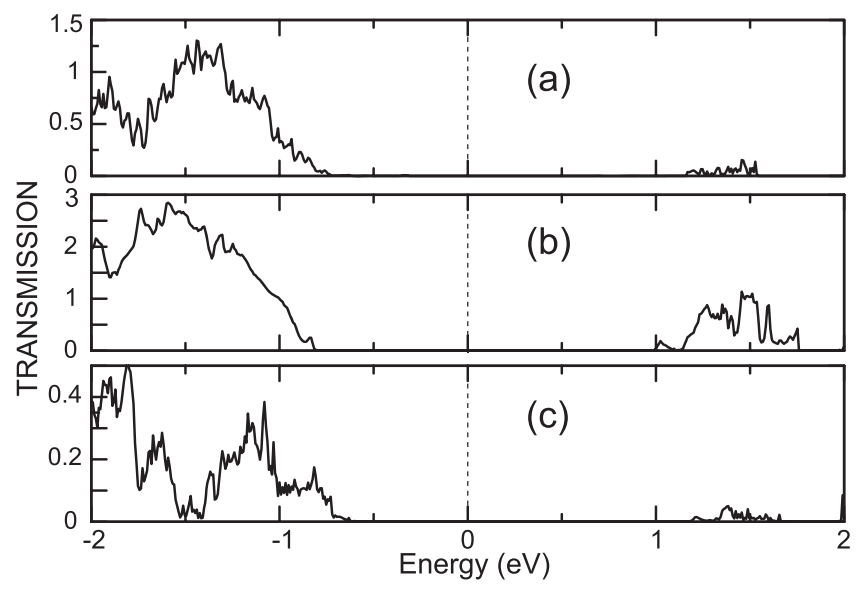

Figure 2. The zero bias transmission spectra for (a) the $\alpha$-device, (b) the $\beta$ device, and (c) the $\gamma$-device.

$\gamma$ structure significantly deviates from planar geometry after structural relaxation, see figure 1 . To check whether such distortion is due to a calculation artifact, we started from a complete planar geometry and allowed both atomic coordinates and cell parameters relax to their equilibrium values (or lowest energy configuration). We observed that planar structure is not stable and structural relaxation brought back the original distorted structure. Indeed, such buckling or deviation from planar structure mainly restricts to the left interface, in line with a recent work that proposed a new crystal structure model for $\mathrm{MoS}_{2}$ [29]. Observed buckling helps to reduce repulsive interaction between $\mathrm{S}$ atoms at the left interface, thereby enhancing the stability of this interface.

The transmission spectra for all three device models at zero bias are depicted in figure 2. In these plots, the Schottky barrier for holes (electrons) is defined as the difference between Fermi level and valence band maximum (conduction band minimum) of the semiconductor $1 \mathrm{H}$ phase of $\mathrm{MoS}_{2}$. The first clear observation is that there is a large barrier height at the pristine interfaces and there is no transmission within an energy range of $1.8 \mathrm{eV}$ around the Fermi level, corresponding to the band gap of $1 \mathrm{H}-\mathrm{MoS}_{2}$. The Schottky barrier heights for the $\alpha, \beta$ and $\gamma$ structures are predicted as $0.72,0.80$, and $0.63 \mathrm{eV}$ for holes and 1.16, 0.99 and $1.19 \mathrm{eV}$ for electrons, respectively. The estimated size of the scattering region along the transport direction is larger than $23 \AA$, which is much smaller than the mean free path of electron in $\mathrm{MoS}_{2}$ [30] and therefore, the transport properties of these systems can be estimated with ballistic transport calculations. The $\beta$ structure has the largest transmission over the calculated energy range. The Mo atoms form a zigzag chain perpendicular to the interface (or along the transport direction) in the $\beta$ and also $\gamma$ structures which enhances the electrical transport in these systems. However, the non-symmetric Mo zigzag chain lying parallel to the transport direction leads to scattering of electrons at the interface and gives rise to low transmission as compared to the $\alpha$ and $\beta$ structures. Similar anisotropic electron transport has also been observed for $\mathrm{ReS}_{2}$ where resistance is the lowest along the Re cluster direction [31]. Comparing the $\alpha$ and $\beta$ devices, the coexistence of $1 \mathrm{~T}$ and 


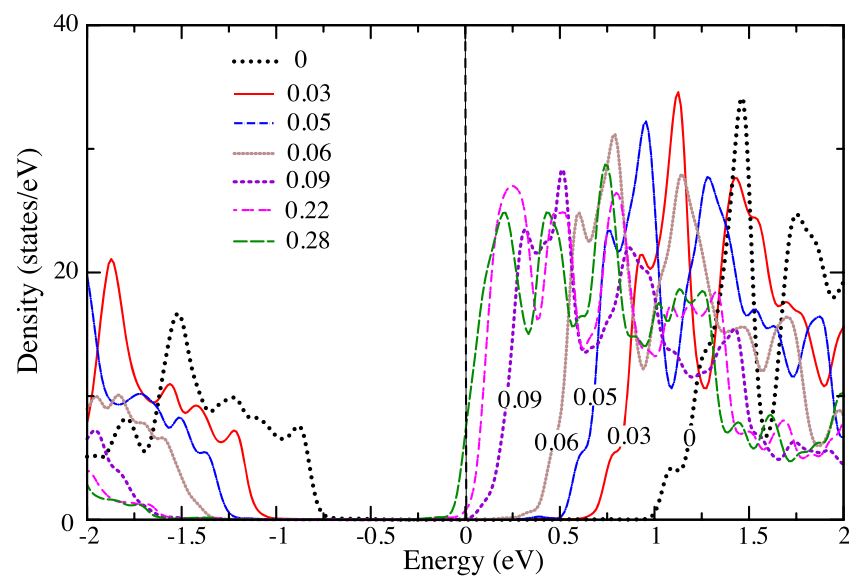

Figure 3. Projected density of states of the valence and conduction band of $1 \mathrm{H}-\mathrm{MoS}_{2}$ as a function of electron concentration for the $\beta$-device. Here, we only show the PDOS of the central part of $1 \mathrm{H}-\mathrm{MoS}_{2}$ where the effect of the interface is minimal. The Fermi level marks the zero energy. Electron concentrations (per formula unit of $1 \mathrm{~T}_{d}$ phase) are given.

$1 \mathrm{~T}_{d}$ regions in the former device contributes to lowering of the transmission due to additional scattering at the $1 \mathrm{~T} / 1 \mathrm{~T}_{d}$ interface as compared to the latter device where we only have $1 \mathrm{~T}_{d}$ phase in the electrode region.

We next turn to the calculations of the electronic properties as a function of doping. The central part of $1 \mathrm{H}-\mathrm{MoS}_{2}$ as the least affected from interface formation is considered to predict the band gap and the position of the band edges with respect to the Fermi level. Figure 3 shows the position of the Fermi level of the $\beta$ structure with respect to the valence and conduction band of the central part of $1 \mathrm{H}-\mathrm{MoS}_{2}$ as a function of electron doping. The first observation is that the calculated band gap value of undoped $\mathrm{MoS}_{2}$ (which is found to be $1.75 \mathrm{eV}$ ) clearly indicates that the size of the $1 \mathrm{H}$ part is large enough to achieve the monolayer limit and eliminates the electrode-electrode interaction. In fact, the band gap of the pristine $1 \mathrm{H}-\mathrm{MoS}_{2}$ monolayer calculated with the same functional is around $1.7 \mathrm{eV}$. In line with the transport calculations, the Fermi level appears within the band gap of the central region of $1 \mathrm{H}-\mathrm{MoS}_{2}$. The calculated Schottky barriers are $0.75 \mathrm{eV}$ for holes and $0.99 \mathrm{eV}$ for electrons in the $\beta$ structure. In the following discussion, we mainly focus on the $\beta$ structure due to its better transport properties as compared to the $\alpha$ and $\gamma$ devices. Other device models also exhibit similar properties. Our results contradicts experimental findings in the sense that, in experiments, it was shown that $1 \mathrm{~T}$ (or $\left.1 \mathrm{~T}_{d}\right) \mid 1 \mathrm{H}-\mathrm{MoS}_{2}$ interfaces exhibit a superior performance over the $3 \mathrm{D}$ metal- $\mathrm{MoS}_{2}$ interfaces. However, we predict large Schottky barriers which give rise to a large contact resistance. In order to shed light on this contradiction, we calculate the electronic properties of the $\beta$ structure as a function of electron doping. First of all, the electron doping stabilizes the $1 \mathrm{~T}_{d}$ phase over the $1 \mathrm{H}-\mathrm{MoS}_{2}$ and prevents the structural phase transition to the semiconducting $1 \mathrm{H}-\mathrm{MoS}_{2}$ phase [32]. Also, the electron doping decreases the Schottky barrier height for electrons at the interface, leading to the formation of $n$-type Schottky barrier. This is attributed to the increase of the

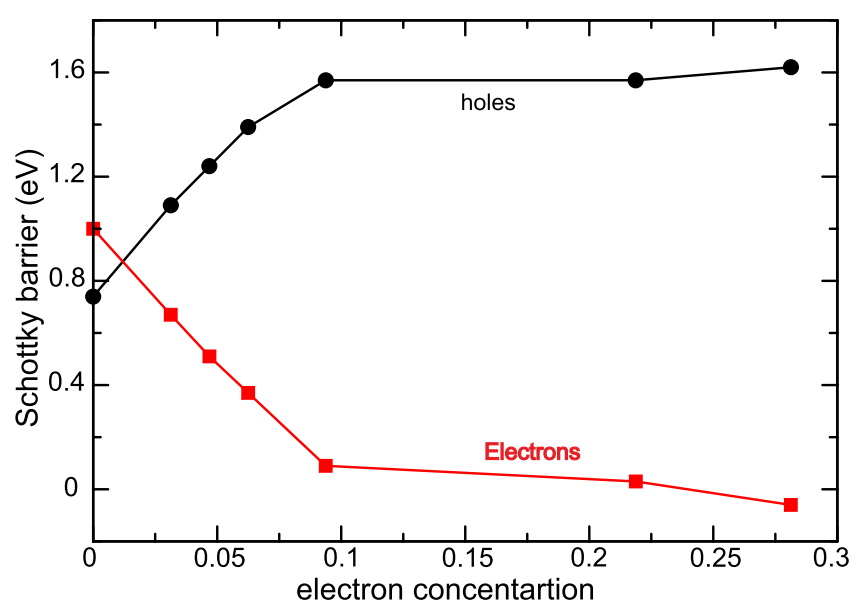

Figure 4. Variation of Schottky barrier for the $\beta$-device as a function of electron concentration (per formula unit of $1 \mathrm{~T}_{d}$ part) for both electrons (red) and holes (black).

density of electrons in the $d$-orbital of the metallic $1 \mathrm{~T}_{d} \mathrm{MoS}_{2}$ phase, see figure 3. Figure 4 shows the variation of the Schottky barrier as a function of the electron concentration. The Schottky barriers are calculated from figure 3. We find that the Schottky barrier already diminishes for electron concentration larger than 0.1 electron (per $1 \mathrm{~T}_{d} \mathrm{MoS}_{2}$ formula unit). Here, 1 electron doping corresponds to a carrier density of $5.5 \times 10^{14} \mathrm{~cm}^{-2}$ using rectangular $1 \mathrm{~T}_{d}$ unit cell. The Fermi level rises about $1 \mathrm{eV}$ when 0.28 electron is placed on the $1 \mathrm{~T}_{d}$ part. While the 0.3 electron doping per formula unit is a high doping limit, we can still achieve a $0.75 \mathrm{eV}$ reduction of the Schottky barrier with a much smaller electron doping such as 0.06 electron per formula unit. Similarly, the Schottky barrier for holes is expected to be reduced by p-type doping (i.e. hole doping). The direct electron doping can be achieved by using electron beams in experiments or $\mathrm{Li} / \mathrm{Na}$ adsorption on the metallic phase $[32,33]$. Here, the considered alkali atoms donate their electron to the $1 \mathrm{~T}_{d}$ phase and enhance the stability and electronic properties of the metallic part [32]. Charge doping lowers the transition barrier and induces a phase transition: $1 \mathrm{H} \rightarrow 1 \mathrm{~T} \rightarrow 1 \mathrm{~T}_{d}$. In addition, absorption of hydrogen atom on the $1 \mathrm{~T}$ part of $\mathrm{MoS}_{2}$ has been also shown to reduce the barrier at the interface of $1 \mathrm{~T}-\mathrm{MoS}_{2} \mid 1 \mathrm{H}-\mathrm{MoS}_{2}$ $[34,35]$. We can also realize either $n$-type or $p$-type doping of metallic phase of $\mathrm{MoS}_{2}$ by using a suitable substrate. For instance, the calculated work function of $1 \mathrm{H}-\mathrm{MoS}_{2}$ is $5.2 \mathrm{eV}$. A low work function metal leads to electron doping in the $1 \mathrm{H}-\mathrm{MoS}_{2}$. As a result, we may have a structural phase transition from $1 \mathrm{H}$ to $1 \mathrm{~T}_{d}$.

In lateral 2D heterostructures, the electronic band alignment is strongly dependent on the heterostructure width even in the absence of polar edges. In contrast to traditional 3D junctions, a highly non-localized charge transfer may be present in the lateral 2D junctions, which considerably increases the junction size [36-40]. Instead of having a narrow transition region between depleted region and neutral zone as in the case of $3 \mathrm{D}$ junctions, the transition region may even extend over entire devices in $2 \mathrm{D}$ junctions due to reduces electronic screening. In this respect, by enlarging the 
$1 \mathrm{H}-\mathrm{MoS}_{2}$ part and vacuum size, we performed test calculations to check the dependence of the Schottky barrier heights on the size of device region for the uncharged and charged system with 0.11 electron/f.u. In the new system, the size of the $1 \mathrm{H}-\mathrm{MoS}_{2}$ part and vacuum size are $57 \AA$ and $20 \AA$, respectively. We found that the Schottky barrier changes by at most $0.05 \mathrm{eV}$.

Another possible strategy to enhance stability of metallic phases and electrical conduction at the metal-semiconductor $\mathrm{MoS}_{2}$ interface is to dope metallic phase with transition metal atoms. Most of the well known TMDCs are either in the $1 \mathrm{H}$ or $1 \mathrm{~T}$ phase when in their ground state. However, the single layer $\mathrm{ReS}_{2}$ has neither $\mathrm{H}$ nor $\mathrm{T}$ as ground state, it stabilizes in $1 \mathrm{~T}_{d}$ structure $[41,42]$. Therefore, alloying $\mathrm{MoS}_{2}$ with Re may stabilize the $1 \mathrm{~T}_{d}$ structure of $\mathrm{MoS}_{2}$ and leads to $n$-type doping of the crystal as similarly proposed by Raffone et al for $\mathrm{Sn}$ doped 1T phase [43]. Meanwhile, we have previously shown that doping of $\mathrm{ReS}_{2}$ with Mo results in a $p$-type doping of $\mathrm{ReS}_{2}$ monolayer [42]. Therefore, we investigated the effect of substitutional doping of Re at Mo sites of $1 \mathrm{~T}_{d}-\mathrm{MoS}_{2}$ on the transport properties. Here, we also considered the group $\mathrm{V}$ element Ta since the pristine $\mathrm{TaS}_{2}$ monolayer crystallizes in the $1 \mathrm{~T}$ phase and results in a $p$-type doped $1 \mathrm{~T}_{d} \mathrm{MoS}_{2}$ structure. Indeed, in a recent work, it was shown that distorted phase of $\mathrm{MoS}_{2}$ becomes energetically stable over $1 \mathrm{H}$ phase when Re concentration exceeds 50\% [44]. In this work, we did not consider such large dopant concentrations because of two reasons. First of all, lattice mismatch between $1 \mathrm{H}-\mathrm{MoS}_{2}$ and doped $1 \mathrm{~T}-\mathrm{MoS}_{2}$ phases can be kept minimal for small dopant concentrations. At large concentrations, the relaxation of cell parameters leads to artificial enlargement of lattice parameters of $1 \mathrm{H}-\mathrm{MoS}_{2}$. Secondly, Re-doped $1 \mathrm{~T}_{d}-\mathrm{MoS}_{2}$ becomes a semiconductor. To show the effect of doping, we only considered concentrations smaller than $20 \%$. In addition, we only considered homogenous distribution of dopant atoms. In this work, we assumed that doping of $1 \mathrm{~T}-\mathrm{MoS}_{2}$ with $\mathrm{Re}$ or Ta may avoid the structural transition to $1 \mathrm{H}$ phase due to, for instance, temperature effect. Figure 5 shows the PDOS for the central part of $1 \mathrm{H}-\mathrm{MoS}_{2}$ for Re and Ta-doped $\beta$ structure. In the case of Re doping, the Fermi level approaches the conduction band of $1 \mathrm{H}-\mathrm{MoS}_{2}$, accompanying a significant decrease in $n$-type Schottky barrier height. On the other hand Ta doping reduces the $p$-type Schottky barrier height as expected. For a concentration of $14 \%$ (per electrode), the $n$-type Schottky barrier becomes $0.85 \mathrm{eV}$ for Re and $p$-type Schottky barrier becomes $0.58 \mathrm{eV}$ for Ta. We also checked the impact of the length of the $1 \mathrm{H}-\mathrm{MoS}_{2}$ part, which is $57 \AA$, on the electronic properties. Similar to previous case, we found that $\operatorname{Re}(\mathrm{Ta})$ doped system has lower Schottky barrier for holes (electrons) as compared to the pristine (i.e. undoped) system.

Since $\mathrm{Re}$ and Ta doping give rise to different electronic properties, we can design metal-semiconductor junctions with different type of Schottky barrier heights (i.e. $n$ - and - $p$ type) in the same device geometry. This allows us to design optical and photovoltaic applications. While a Re-doped junction effectively blocks holes, Ta-doped junction hampers the easy passage of electrons across the junction. In this device

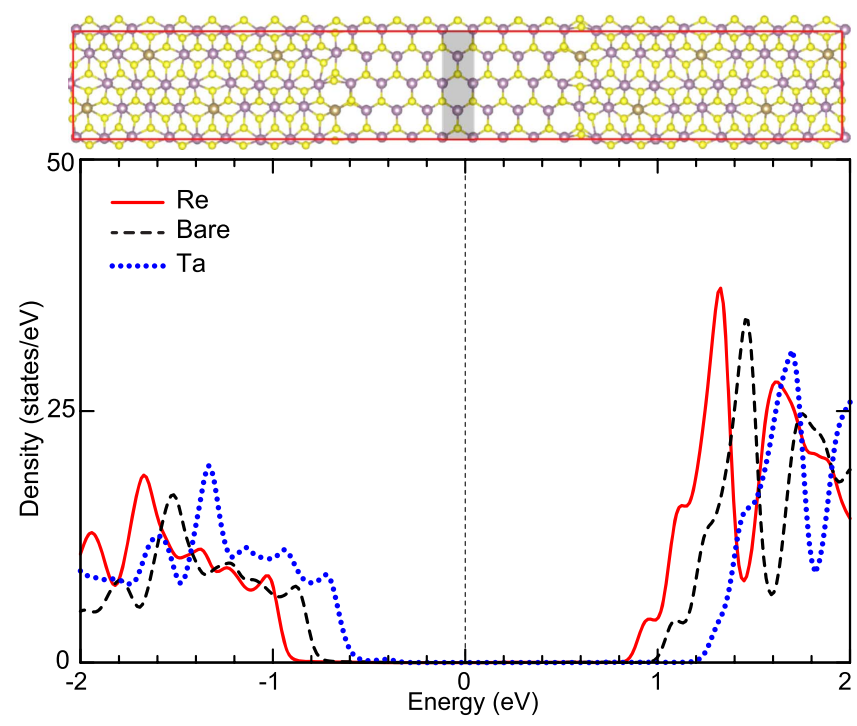

Figure 5. Projected density of states of the valence and conduction band of the central part of $1 \mathrm{H}-\mathrm{MoS}_{2}$ for Re and Ta-doped devices. In the top figure, gray region highlights the central part of $1 \mathrm{H}$ phase for which PDOS is calculated. For comparison, PDOS of bare device is also shown.

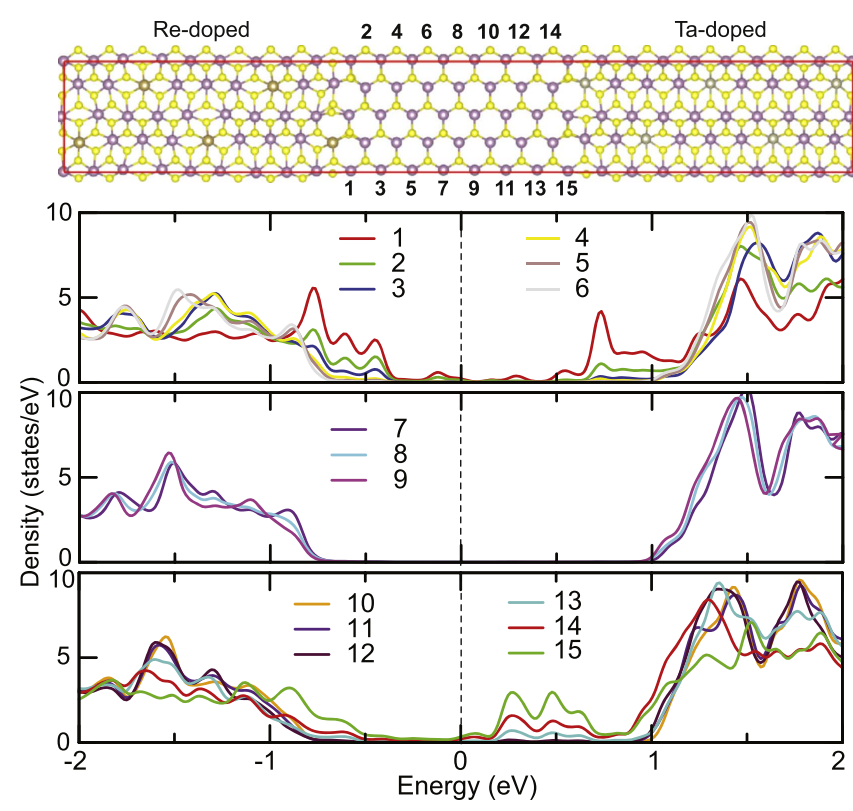

Figure 6. Projected density of states of $1 \mathrm{H}-\mathrm{MoS}_{2}$ at the different position on $1 \mathrm{H}-\mathrm{MoS}_{2}$. The Fermi level marks the zero energy.

geometry, we can separate photo-generated charge carriers for instance. Figure 6 shows the device model and projected density of states as a function of position in $1 \mathrm{H}-\mathrm{MoS}_{2}$. While the left electrode is doped with $\mathrm{Re}$, the right electrode is alloyed with Ta. The central part of $1 \mathrm{H}-\mathrm{MoS}_{2}$ clearly has a PDOS similar to free standing $1 \mathrm{H}-\mathrm{MoS}_{2}$ monolayer with a band gap of $1.75 \mathrm{eV}$. However, we have different electronic properties in the right and left side of the central region. Due to $\operatorname{Re}(\mathrm{Ta})$ doping, the left (right) part has a $n(p)$-type Schottky barrier. The presence of $1 \mathrm{~T}_{d}-1 \mathrm{H}-\mathrm{MoS}_{2}$ interfaces develops mid-gap states that mainly come from the atoms in the boundary region. The electronic properties gradually 


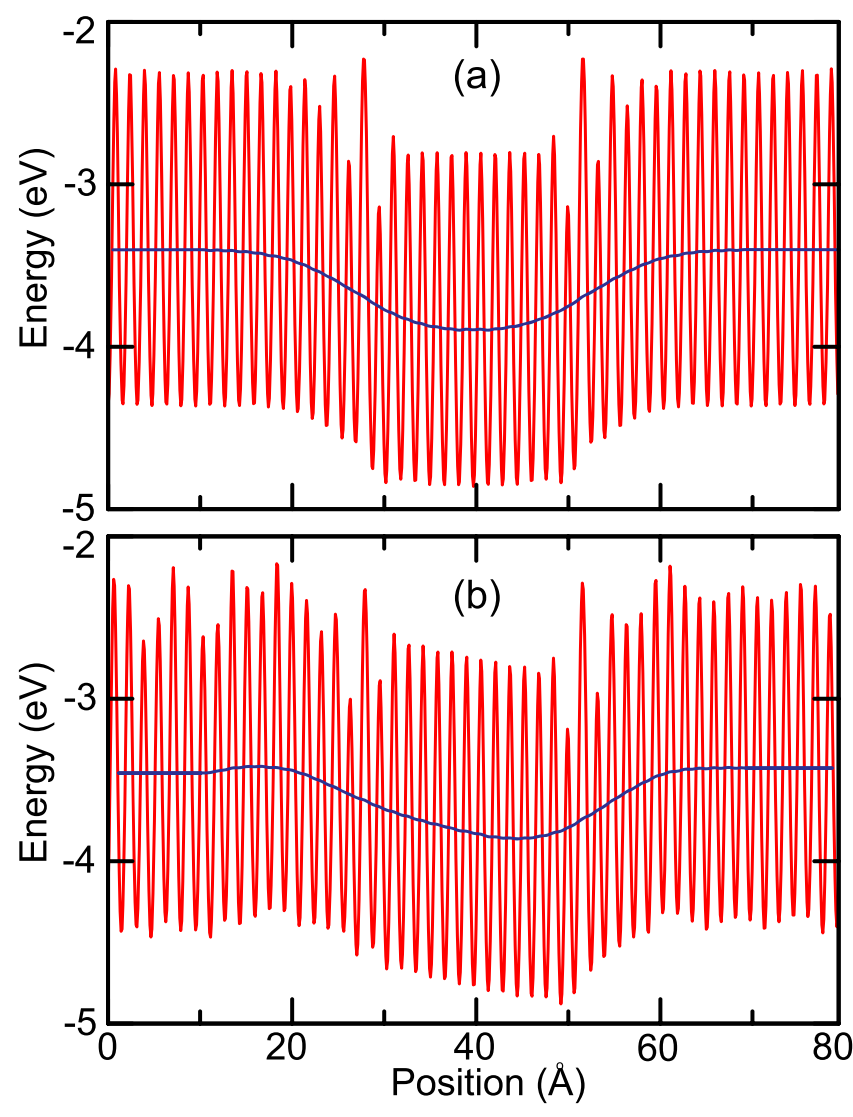

Figure 7. Self-consistent electrostatic potential profile along the interface of armchair (a) pristine and (b) doped $1 \mathrm{~T}_{d}-1 \mathrm{H}-1 \mathrm{~T}_{d} \mathrm{MoS}_{2}$ heterostructure. The right (left) $1 \mathrm{~T}_{d} \mathrm{MoS}_{2}$ is doped with Re (Ta). Blue curve denotes the plane average potential along the heterostructure.

change from the metallic to the semiconductor when moving away from the interfaces. For the atoms far away from the interface region (i.e. central region of $1 \mathrm{H}-\mathrm{MoS}_{2}$ ), we observe a clear band gap which is close to that of pristine $1 \mathrm{H}-\mathrm{MoS}_{2}$. While the mid-gap states appear below the Fermi level at the left interface (Re-doped side), they are unoccupied and reside above the Fermi level at the right interface (Ta-doped side). About $3.2 \AA$ from the interface, the mid-gap states start to disappear.

Figure 7 shows the electrostatic potential along the heterojunction. We consider both pristine and doped $\beta$-devices. For undoped heterojunction, the average potential is symmetric at the left and right interfaces. However, doped heterojunction has a different electrostatic potential, especially, within $1 \mathrm{H}-\mathrm{MoS}_{2}$. Due to its valence configuration, Re (Ta) acts as a donor (an acceptor). This is reflected in the average effective potential shown in figure 7(b). The average electrostatic potential (EP) does not have a sharp variation at the $1 \mathrm{~T}_{d^{-}} 1 \mathrm{H}$ interface, extending along the $2-3$ atomic rows. This is due to fact that we form interfaces between two different crystal structures of $\mathrm{MoS}_{2}$ (i.e $1 \mathrm{~T}_{d}$ and $1 \mathrm{H}$ ). EP converges to the same value at the left and right electrodes. If one considers a photovoltaic device using the $\beta$ structure co-doped with $\operatorname{Re}$ and $\mathrm{Ta}$, an electron-hole pair is generated after absorbing a photon in the $1 \mathrm{H}$ part. Re-doped interface has a higher potential as compared to Ta-doped interface, producing a driving force for dissociation of the electron-hole pair. The electron flows along the potential decline (i.e. towards Tadoped electrode) and the hole in the opposite direction (i.e. towards Re-doped electrode). In this way, a photocurrent can be generated by the photovoltaic effect. Thus, by proper control of doping and interface roughness, we can control the quantum efficiency of electron-hole dissociation [45].

\section{Conclusion}

In this work, we explored the impact of doping on the electronic and charge transport properties across the $1 \mathrm{~T}_{d}-1 \mathrm{H}-\mathrm{MoS}_{2}$ interfaces by considering various device models. Doping and alloying (with charge, atom or molecule) appear as an effective method to tailor and improve the physical-chemical properties and stabilities of not only $1 \mathrm{~T} / 1 \mathrm{~T}_{d}$ phases of $\mathrm{MoS}_{2}$ but also other $2 \mathrm{D}$ materials. The interface structure between $1 \mathrm{~T}_{d}$ and $1 \mathrm{H}$ phases is one of the decisive factors in the determination of the electrical transport across the heterojunction. We found that the Schottky barrier height of electrons for pristine heterojunctions can even disappear as a result of electron doping. While charge doping only reduces the Schottky barrier for electrons, codoping is able to tune the barriers for hole and electrons at the same time.

\section{Acknowledgments}

This work was supported by the bilateral project between the The Scientific and Technological Research Council of Turkey (TUBITAK) and FWO-Flanders, Flemish Science Foundation (FWO-Vl) and the Methusalem foundation of the Flemish government. Computational resources were provided by TUBITAK ULAKBIM, High Performance and Grid Computing Center (TRGrid e-Infrastructure), and HPC infrastructure of the University of Antwerp (CalcUA) a division of the Flemish Supercomputer Center (VSC), which is funded by the Hercules foundation. We acknowledge the support from TUBITAK (Grant No. 115F024).

\section{ORCID iDs}

Deniz Çakır (iD https://orcid.org/0000-0003-3315-5204

\section{References}

[1] Singh J 1993 Physics of Semiconductors and Their Heterostructures (Electrical Engineering Series) (New York: McGraw-Hill)

[2] Agostini G and Lamberti C 2011 Characterization of Semiconductor Heterostructures and Nanostructures (Amsterdam: Elsevier) 
[3] Gan L-Y, Zhang Q, Cheng Y and Schwingenschlögl U 2013 Phys. Rev. B $\mathbf{8 8} 235310$

[4] Wu N, Yang Z, Zhou W, Zou H, Xiong X, Chen Y and Ouyang F 2015 J. Appl. Phys. 118084306

[5] Bartolomeo A D 2016 Phys. Rep. 6061

[6] Duerloo K-A N and Reed E J 2016 ACS Nano 10289

[7] Allain A, Kang J, Banerjee K and Kis A 2015 Nat. Mater. 141195

[8] Matsuda Y, Deng W-Q and Goddard W A 2010 J. Phys. Chem. C 11417845

[9] Kang J, Liu W, Sarkar D, Jena D and Banerjee K 2014 Phys. Rev. X 4031005

[10] Eda G, Fujita T, Yamaguchi H, Voiry D, Chen M and Chhowalla M 2012 ACS Nano 67311

[11] Kappera R, Voiry D, Yalcin S E, Branch B, Gupta G, Mohite A D and Chhowalla M 2014 Nat. Mater. 131128

[12] Fan X, Xu P, Zhou D, Sun Y, Li Y C, Nguyen M A T, Terrones M and Mallouk T E 2015 Nano Lett. 155956

[13] Huang C, Wu S, Sanchez A M, Peters J J P, Beanland R, Ross J S, Rivera P, Yao W, Cobden D H and Xu X 2014 Nat. Mater. 131096

[14] Zhang X-Q, Lin C-H, Tseng Y-W, Huang K-H and Lee Y-H 2015 Nano Lett. 15410

[15] Bai Z, Markussen T and Thygesen K S 2013 arXiv:1311.2393

[16] Hu Z, Zhang S, Zhang Y-N, Wang D, Zeng H and Liu L-M 2015 Phys. Chem. Chem. Phys. 171099

[17] Sivaraman G, de Souza F A L, Amorim R G, Scopel W L, Fyta M and Scheicher R H 2016 J. Phys. Chem. C 120 23389

[18] Kresse G and Hafner J 1993 Phys. Rev. B 47558

[19] Kresse G and Hafner J 1994 Phys. Rev. B 4914251

[20] Kresse G and Furthmüller J 1996 Comput. Mater. Sci. 615

[21] Kresse G and Furthmüller J 1996 Phys. Rev. B 5411169

[22] Blöchl P E 1994 Phys. Rev. B 5017953

[23] Kresse G and Joubert D 1999 Phys. Rev. B 591758

[24] Perdew J P, Burke K and Ernzerhof M 1996 Phys. Rev. Lett. 773865
[25] Perdew J P, Burke K and Ernzerhof M 1997 Phys. Rev. Lett. 781396

[26] Brandbyge M, Mozos J-L, Ordejón P, Taylor J and Stokbro K 2002 Phys. Rev. B 65165401

[27] Soler J M, Artacho E, Gale J D, Garcia A, Junquera J, Ordejón P and Sanchez-Portal D 2002 J. Phys.: Condens. Matter 142745

[28] Troullier N and Martins J L 1991 Phys. Rev. B 431993

[29] Ma F, Gao G, Jiao Y, Gu Y, Bilic A, Zhang H, Chen Z and Du A 2016 Nanoscale 84969

[30] Radisavljevic B, Radenovic A, Brivio J, Giacometti V and Kis A 2011 Nat Nanotechnol. 6147

[31] Lin Y-C, Komsa H-P, Yeh C-H, Bjrkman T, Liang Z-Y, Ho C-H, Huang Y-S, Chiu P-W, Krasheninnikov A V and Suenaga K 2015 ACS Nano 911249

[32] Kan M, Wang J Y, Li X W, Zhang S H, Li Y W, Kawazoe Y, Sun Q and Jena P 2014 J. Phys. Chem. C 1181515

[33] Katagiri Y et al 2016 Nano Lett. 163788

[34] Nourbakhsh A et al 2016 Nano Lett. 167798

[35] Tang Q and Jiang D-E 2015 Chem. Mater. 273743

[36] Leenaerts O, Vercauteren S and Partoens B 2017 Appl. Phys. Lett. 110181602

[37] Zhang J, Xie W, Zhao J and Zhang S 2017 2D Mater. 4 015038

[38] Yu H, Kutana A and Yakobson B I 2016 Nano Lett. 165032

[39] Cheng K, Guo Y, Han N, Su Y, Zhang J and Zhao J 2017 J. Mater. Chem. C 53788

[40] Leenaerts O, Vercauteren S, Schoeters B and Partoens B 2016 2D Mater. 3025012

[41] Tongay S et al 2014 Nat. Commun. 53252

[42] Cakir D, Sahin H and Peeters F M 2014 Phys. Chem. Chem. Phys. 1616771

[43] Raffone F, Ataca C, Grossman J C and Cicero G 2016 J. Phys. Chem. Lett. 72304

[44] Tan T L, Ng M-F and Eda G 2016 J. Phys. Chem. C 1202501

[45] Cao Z, Harb M, Lardhi S and Cavallo L J. Phys Chem. Lett. 8 1664 pMID: 28332394 